\begin{abstract}
Introduction: This study examined types of reported simultaneous mixed emotion experiences for the first time in adolescence for high and low intensity emotion pairs using an Analogue Emotion Scale which affords the graphing of two opposite valence emotions over time on the same graph. Methods: In a cross sectional design, 163 participants based in schools across the UK formed two age groups representing early and mid-adolescence (12 years, 5 months-16 years, 9 months vs. 16 years, 10 months-18 years, 8 months) across two conditions considering either their own $(n=83)$ or another child's $(n=80)$ emotional experience divided equally for high $(n=80)$ or low $(n=83)$ intensity mixed emotion pairs presented in vignettes about themselves or another person. They were seen individually and completed an emotion presence interview and an Analogue Emotion Scale about the emotions experienced in the condition appropriate vignette. Results: Participants reported mixed emotions both sequentially and simultaneously. In particular we found that children showed that others experience emotions in a more sequential manner, while they themselves would experience more emotions in a highly simultaneous way. Emotion experience was different depending on the emotion pair and age group. Conclusions: Adolescents' subjective mixed emotion experiences vary by intensity and patterns of simultaneity over time. Findings are discussed in relation to an evaluative space model of mixed emotion and applications of the AES with adolescent populations.
\end{abstract}

Keywords: Mixed emotion; adolescence; self; other. 


\section{Mixed emotion experiences for self or another person in adolescence Introduction}

\section{Types of subjective mixed emotion experiences}

The present study assessed reported subjective mixed emotional experiences for low and high intensity emotion pairs across early to mid-adolescence. Mixed emotion experiences are defined as the co-activation or synchrony of two emotions, typically of opposite valence (Carrera \& Oceja, 2007; Larsen, McGraw, \& Cacioppo, 2001).

Evidence is mounting that there is co-activation of opposite valence emotion in adulthood and in mid to late childhood. Mixed emotion can be conceptualised and simultaneously experienced in different ways (Berrios, Totterdell, \& Kellett, 2015; Larsen \& McGraw, 2014; Larsen et al., 2001) and there are two key models for how mixed emotion is experienced. The Circumplex Model (CM) (Russell \& Carroll, 1999) contends that only polar opposite emotions (e.g., happiness and sadness) are mutually exclusive and that emotional experiences with high arousal co-exist, for example, when someone feels stressed and excited at the same time. Alternatively, the Evaluative Space Model (ESM)

posits that the affect system is defined by different operating characteristics for positive and negative emotion that can lead to different behaviours, and are each activated differently within the nervous system (Norris, Gollan, Berntson, \& Cacioppo, 2010). Thus, the ESM is based on the idea that the operation of positive and negative substrates of valence can cooccur and are experientially separable (e.g., Cacioppo, Gardner, \& Berntson, 1999). According to the ESM any pair of opposite valence emotions can be aroused and co-occur, 
including those of high (e.g., angry and excited) and low arousal (e.g., relaxed and tired; Larsen et al., 2001).

Two recent large-scale reviews support the ESM. Larsen and McGraw (2014) found strong evidence in support of the ESM and suggest that a range of emotions with opposite valence and varied intensity of arousal can be experienced simultaneously. Berrios et al. (2015) found that mixed emotion experiences occur beyond the types of emotion pairs and dimensional or discrete nature of the conceptualisation of emotion. They suggested that the affective system could be flexible enough to permit multiple activation patterns of mixed emotion, ranging from bipolar responses, blended emotional responses of mixed similar valence emotion, and different patterns of mixed opposite valence emotions. They highlighted that a pressing question is whether the experience constitutes a genuine coactivation of opposite valence emotions which may be of high or low arousal pairs or a rapid alternating succession between emotions which could be accounted for by both the ESM and CM models. The basis of reported simultaneous types of experience could indeed occur at the level of activation of the affective nervous system or as a consequence of higher reflective processing involved in the process of linguistic or visual representations (Pons, Harris, \& de Rosnay, 2004). Consequently, in relation to the focus of our study, both low and high arousal opposite valence emotion pairs consistent with the ESM may be available for reports of the subjective experiences of types of mixed emotion.

\section{Subjective mixed emotion experiences in childhood and early adolescence}

It is well documented in childhood that mixed emotion understanding in others tends to precede understanding of mixed emotions in the self (Smith, Glass, \& Fireman, 
2015). This mixed emotion recognition in other people and within their own experience increases between 7-12 years (Wintre \& Vallance, 1994). This develops alongside enhanced understanding that mixed emotions can result from increasingly complex emotional situations (Smith et al., 2015). Mixed emotion understanding is argued to first be understood by children in their guardians and peers and later understood in themselves (Heubeck, Butcher, Thorneywork, \& Wood, 2015).

Pons and colleagues' (2004) model describes the nature, causes, and regulation of emotion through three phases, wherein external, internal, and then reflective influences on emotional experience are understood. Progress through the phases is necessarily conditional upon emotional comprehension in the preceding phase. They argued that mixed emotion understanding develops around 8 to 9 years of age. Of interest here is the progression of phases, supported by Rocha et al. (2015) who found a rapid progression through the external (3-6 years), internal (5-9 years), and reflective (8-11 years) phases. These studies do not account for temporal dimensions of mixed emotion experiences, yet they do provide consensus that reflective mixed emotion understanding tends to emerge by 9-11 years, leading into early adolescence. The present study therefore examined the progression of reported types of subjective mixed emotion experiences across early to mid-adolescence which has not previously been assessed in this way.

Adolescents experience significant physiological, emotional, social, and cognitive developmental changes throughout their school years (Larson \& Brown, 2007) and are likely to experience mixed emotions on a daily basis. Their emotional development is often characterised as a wave like pattern of successively fluctuating emotions, often represented 
more by expressions of negative rather than positive emotion (Larson \& Brown, 2007). They tend to report more negative emotional states from early to mid-adolescence and experience more intense emotions in both positive and negative domains than children and adults (Larson et al., 2002). Changes in neurotransmitter levels characteristic across this developmental period can make individuals more sensitive to stress than younger and older populations (Wahlstrom, Collins, White, \& Luciana, 2010). Through the development of abstract reasoning and metacognitive abilities within increasingly complex social interactions (Larson \& Sheeber, 2008), adolescents develop cognitive and affective coping strategies to regulate and express their feelings. These strategies can include ways to minimise negative feelings in order to maintain a positive feeling (Larson \& Sheeber, 2008).

It is reasonable to suggest that during a developmental time of rapid emotional development and increasing exposure to social contexts (Heubeck et al., 2015) that the subjective experiences of mixed emotion, especially those in relation to fluctuations of intensity of emotion (Larson \& Sheeber, 2008) will vary with age. Adolescence is therefore a key time to explore developments in the understanding of mixed emotion experiences in the self and other.

Carrera and Oceja (2007) developed an Analogical Emotion Scale (AES) to measure types of simultaneous experiences of mixed emotion in adulthood. The AES facilitates measurement of the subjective experience of two emotions over the duration of a mixed emotion experience: participants represent two different emotions separately on the same graph with two axes, one axis represents emotion intensity and the other emotion 
duration. They used the AES, alongside emotion rating scales, to understand undergraduates' experiences of co-occurring happiness and sadness through the recollection of an everyday situation. Importantly, they found that mixed emotions were over-reported using the scales relative to the AES responses and argued that asking participants to generate a numerical value overestimates the presence of mixed emotions and does not identify temporal changes across the experience. To establish and validate the AES, Oceja and Carrera (2009) conducted three further studies and analysed mixed emotional experiences across the above and additional three studies using different mixed emotion eliciting methods. In their second study three scenarios about short emotional experiences were used showing more mixed emotions than simple emotions. In two further studies, where the structure of the mixed happy and sad experiences was manipulated, and where pictures were used to elicit mixed emotion, more simultaneous than sequential experiences were found.

They found that the intensity of the emotions moderated simultaneous mixed emotion experiences across the four studies. All studies showed that the presence of types of simultaneous mixed emotion experiences with different kinds of experience depending on how the intensity of the emotions changed over time was greater in the mixed than the simple conditions. They identified four patterns of mixed emotion experience (see Figure $1)$.

\section{**PLEASE INSERT FIGURE 1 ABOUT HERE**}

Firstly, the sequential pattern where one emotion is present but then replaced by a second emotion. Secondly, the prevalence pattern where both emotions are enduring but 
one is of a higher intensity and the other of a much lower intensity. Thirdly, an inverse pattern where both emotions are present but one increases and the other decreases over time. Finally, the highly simultaneous pattern where both emotions are present at moderate to high intensity with one slightly higher in intensity than the other throughout the experience.

There is tentative evidence of the same patterns of subjective mixed emotion in mid childhood (Burkitt, Lowry, \& Fotheringham, 2017) as categorised in adult populations (Carrera \& Oceja, 2007). From 6 years old, children report that the experience of opposite valence emotion is sequential rather than simultaneous (e.g., Wintre \& Vallance, 1994). Yet, using an AES, Burkitt et al. (2017) showed that 4- to 6-year-olds can report all four types of mixed emotion experiences, with the older children reporting a greater number of simultaneous experiences. Interestingly, older children reported more prevalence experiences than young children when considering their own experiences.

\section{High and low arousal mixed emotions pairs}

It is accepted that high arousal emotions (e.g., stress and excitement) can co-occur during an emotional experience (Russell \& Carrol, 1999), however less is known about the co-occurrence of emotions with low arousal (e.g., tired and relaxed). Larsen \& McGraw (2014) called for the examination of lower arousal emotion pairs such as relaxation and tiredness. It may be that varying the types of emotion by arousal intensity may impact the likelihood of experiencing mixed emotion rather than simply the emotional positive and negative valence within an experience (Rafaeli, Rogers, \& Revelle, 2007). It may also be the case that more intense experiences of simultaneous mixed emotions will be apparent for 
high rather than low arsousal emotions. Patterns of simultaneous mixed emotions identified in adulthood and middle childhood (e.g., Burkitt et al., 2017; Ojeca and Carrera, 2009) that vary by intensity over the course of an event have not been examined in relation to both high and low arousal emotion pairs. The present study therefore compared reported high and low arousal emotion pairs to assess whether the type of emotion pair impacted on how the subjective mixed emotion was experienced.

\begin{abstract}
Aims
Our primary aim was to examine the presence of patterns of subjective mixed emotion experiences across early to mid-adolescence. On the basis of COjeca \& Carrera's (2009) work, it was anticipated that sequential, prevalence, inverse, and highly simultaneous categories would be identified. We investigated if these types would be impacted by emotion pairs of varying arousal (happy/sad versus relaxed/ tired). We expected, in line with Ojeca and Carrera (2009), that adolescents will report more highly simultaneous experiences for the high than the low arousal emotion pair. Given that mixed emotions may be perceived differently in regard to the experiences of the self or other (Burkitt et al., 2017; Heubeck et al., 2015) we explored whether mixed emotion experiences would be reported differently if they were in reference to the self or another person. Importantly, as older adolescents may have stronger understanding of complex emotional experiences, we expected more complex rather than sequential types of experiences to be reported in mid than in early adolescence.
\end{abstract}

\title{
Method
}




\section{Participants}

Following approval from the host University Research Ethics Committee, participants $(N=163 ; 80$ females $)$ were recruited from three secondary schools in West Sussex and Hampshire, UK which had comparable middle to high indices of social economic status. The sample was split into two age groups of equivalent numbers to reflect the periods of early and mid-adolescence where negative emotionality and greater intensity of both positive and negative emotions are increasingly experienced (Larson et al., 2002): early adolescence group ( $n=81$, range $=12$ years 5 months to16 years 9 months $)$ and a midadolescence group ( $n=82$, range $=16$ years 10 months to 18 years 8 months). Within each age group participants were randomly allocated (in approximately equal numbers, see Table 1) to four conditions: self-experience with high intensity, self-experience with low intensity, other-experience with high intensity, or other-experience with low intensity. The sample size was calculated as sufficient for the present design (Faul, Erdfelder, Lang, \& Buchner, 2007).

\section{Materials}

Two emotion pair vignettes were developed describing a situation where happy and sad emotions would be experienced or relaxed and tired emotions would be experienced. For each emotion pair vignette there were two versions, one with the self being the focus of the emotion experience and one with the focus of emotion experience being another child (please see Appendix A for the vignettes). The happiness and sadness vignette was selected from previous research (Burkitt et al., 2017). The relaxed and tired vignette was developed based on Larsen and McGraw's (2014) suggestion to study the emotion pairing 
and matched in length and number of affective episodes to the happiness and sadness vignette $^{1}$. The AES graphs were adapted from Ojeca and Carrera's (2009) research (see example in Appendix B) to assess sequential, prevalence, inverse, and highly simultaneous types of mixed emotion patterns where the experience of each emotion varies by intensity across the duration of the experience. The axes labels were simplified from the original graph to ask how long the experience lasted to measure duration on the $\mathrm{x}$ axis, and to ask how much the feeling was felt to measure intensity on the y axis. This version has been used in previous research with younger populations (Burkitt et al., 2017). The AES has been found to reliably discriminate between sequential and simultaneous mixed emotions (Ojeca \& Carrera, 2009; Carrera \& Ojeca, 2007) and between types of simultaneous mixed emotions (Burkitt et al., 2017; Ojeca \& Carrera, 2009) which vary by intensity and temporal pattern in middle childhood and in adulthood. Studies have shown the validity of the scale in detecting temporal patterns of mixed emotion experiences that mirror the valence and temporal flow of events in stimuli designed to elicit opposite valence emotional response across a specific duration (Ojeca \& Carrera, 2009).

\section{Procedure}

${ }^{1}$ The relaxed and tired self and other vignettes were piloted for comprehension with seven 12-year-olds and eight 17-year-olds for the self-vignette and eight 12-years-olds and eight 17-year-olds for the other-vignette. They were asked how the episodes made them feel and to produce corresponding AES responses. The vignettes discriminated temporal trajectories and intensity levels between relaxed and tired emotion responses. Participants were lastly asked if the vignettes made sense and attested to the comprehensibility the text. 
Participants completed the testing individually in their normal classroom within sight of the class teacher. They read the condition (self or other) appropriate vignette relating to either high or low intensity emotion pairs, and were asked the following emotion presence questions to check understanding of the presence of the intended mixed emotions arising from the vignettes:

"How did you / Alex feel at the end of the story?"

"Did you / Alex feel any other emotion/s at the end?"

Participants were then asked to complete an AES graph about the happy and sad or relaxing and tiring mixed emotion experience. They were asked to think about their condition appropriate (own or Alex's) emotional experience reflecting at the end of the vignette on the events and to graph where the happy / sad or relaxed / tired condition appropriate words were included and their own or Alex's feelings were referred to. It was explained what a line and a dotted line represented in terms of happy, sad, relaxed or tired feelings.

\section{Coding}

The responses to the emotion presence questions were coded by the lead researcher and a second female coder blind to the aims of the study as correctly identifying happy and sad or relaxed and tired experiences when participants used those terms in response across both questions. When participants on occasion (3\%) used more colloquial terms observed in the relaxed and tired condition, such as chilled, knackered or exhausted, inclusion of these terms was discussed by the two coders. The discrepancy of terminology in the relaxed 
and tired condition was resolved by discussion and the responses were included in the subsequent analysis. A mutually exclusive and exhaustive coding process was applied to the AES graphs for all participants who identified the target emotion pairs using Oceja and Carrera's (2009) coding categories of mixed emotion experiences (prevalence, sequential, inverse, and highly simultaneous) by another female researcher naïve to the aims of the research. Any instances outside of this scheme were coded as "unknown". A second trained independent female coder who was naïve to the aims of the study coded $80 \%$ of the graphs. Inter-rater reliability, adjusted for chance agreement, was very good, Cohen's Kappa, $K=0.94$. The discrepancies were resolved through discussion reaching $100 \%$ agreement. One respondent produced an unknown mixed emotion type categorised as "unknown" where the lines both went up and off, and not along, the graph.

\section{Results}

\section{Mixed emotion presence}

Responses to the questions asking about the presence of one or two and which kinds of emotions showed that 158/163 (97\%) participants reported the anticipated two emotions appropriately by condition. The responses of the remaining four participants were removed from subsequent analysis.

\section{Mixed emotion types by condition, emotion pair and age group}

The amount and percentage of mixed emotion response types to the AES within condition, emotion pairing and age group are displayed in Table 1. 
**INSERT TABLE 1 ABOUT HERE**.

Model selection log-linear analysis applying backward elimination was used to explore any main and interaction effects of the adolescent age group (younger versus older), protagonist (self versus other) and emotion pair (high versus low arousal) separately for each graphed emotion experience (sequential, prevalent, inverse and highly simultaneous coded as either absent or present). Log-linear analyses were conducted to estimate the single parameter estimates for the resultant models from the hierarchical model selection procedures. Note that for the prevalence experiences no significant main or interaction models were found. The main and interaction effects are shown in Table 2.

**INSERT TABLE 2 ABOUT HERE**

For sequential types of mixed emotion experience there was a best fit main effect model, partial $\chi^{2}(1)=9.71, p<.001$, of protagonist, with the parameter estimate $\chi^{2}(3)=$ $1.17, p=0.22$, showing more experiences of this when the protagonist was another person $(z=1.65, \mathrm{p}<.001)$ than themselves in part addressing the aim to explore whether reported experiences vary by protagonist. In contrast to sequential mixed emotion experiences for inverse experiences, a main model effect of protagonist, partial $\chi^{2}(1)=11.04, p=<.001$, with the parameter estimate, $\chi^{2}(1)=1.69, p=0.20$, showed that these experiences were more likely to be graphed more by adolescents considering themselves $(z=1.31, \mathrm{p}<.001)$ than another person.

For highly simultaneous mixed emotion experiences there was a main model effect, partial $\chi^{2}(1)=9.54, p=0.01$, of protagonist, with the parameter estimate, $\chi^{2}(1)=1.93, p=$ 
0.30 , showing that this response was graphed more when the self $(z=1.04, p=.02)$ rather than when another person was the protagonist. Further, a main model effect of age group was found, partial $\left.\chi^{2}(1)=4.93, p<.001\right)$ with the parameter estimate, $\chi^{2}(1)=4.19, p=$ .62 , showing that graphed simultaneous experiences were more apparent in mid than early adolescence $(z=1.55, p=.03)$ in line with expectations. According to expectations, a final main model effect of emotion pair was found, partial $\chi^{2}(1)=3.99, p<.001$, with the parameter estimate, $\chi^{2}(1)=4.03, p<.52$, showing that there were more highly simultaneous experiences for the happy sad emotion pair $(z=1.55, p=.03)$. An interaction between age group and emotion pair was significant in the final model, partial $\chi^{2}(1)=$ $3.13, p=0.02$, with single parameter estimate, $\chi^{2}(4)=3.93, p=0.20$, in partial line with expectations indicating that more highly simultaneous experiences for the happy and sad emotions were graphed in mid than early adolescence $(z=1.37, p=.04)$. No further significant main or interaction models emerged for this experience.

\section{Discussion}

The main aim of the present study was to assess the presence of types (Ojeca \& Carrera, 2009) of reported subjective mixed emotion patterns across early to midadolescence We investigated the impact of emotion pairs of varying arousal, protagonist, and age group on mixed emotion experience. Specifically, we anticipated that highly simultaneous types of experiences would result from the higher arousal emotion pair, explored whether responses would vary by consideration of the experiences of self or other and examined the possibility that more complex patterns of mixed emotion experiences would be more evident in mid than early adolescence. 
The four patterns applying to adult populations of sequential, prevalent, inverse, and highly simultaneous experiences were found across this age range (Oceja \& Carrera, 2009). The findings support the Evaluative Space Model where opposite valence emotions of varying arousal are accounted for (Cacioppo et al., 1999; Larsen \& McGraw, 2014; Norris et al., 2010). Reflective mixed emotion understanding (e.g., Pons et al., 2004) is considered to have emerged by early adolescence. This is the first study to demonstrate the occurrence of types of subjective sequential and simultaneous mixed emotion experience for this age range. It also showed that adolescents differ in their perceptions of mixed emotion experiences for themselves and others and, that older adolescents are more likely to differentiate the experiences by the $t$ low or high arousal of the emotion pair.

Effects of considering the experiences of oneself or another person were identified. This work demonstrated that the recognition and understanding of precise types of simultaneous mixed emotion experiences are better understood by adolescents in themselves rather than in other people. For example, more inverse and highly simultaneous responses were graphed by participants in the self compared to the other condition. It is possible that differences between graphed mixed emotional types of responses between the self and the other could be a difference of interpretation between how oneself and other people experience those emotions. For example, someone in adolescence may feel conflicted emotions about an event yet may not realise that another person is experiencing the conflict at all or in the same way. It could also be that individuals are more likely to anticipate more intense emotional experiences in themselves than in others due to a more direct relationship with their own experiences (Grossman \& Wood, 2003). Moreover, 
information that could help us to recognise types of mixed emotion experiences including co-occurrence for types of intensity are directly more evident in the self from signals of emotional arousal and other markers of biofeedback (Cacioppo et al., 1999; Fang, Sauter \& Van Kleef, 2017) and this is information that is unavailable about the emotional experience of others. These intense external and/or internal drivers may influence the subtle kinds of simultaneous mixed emotions they experience and could facilitate understanding in self before others for specific experiences.

As expected, high and low arousal emotion pairs had an impact on highly simultaneous mixed emotion experiences. There were more highly simultaneous mixed emotion experiences for the high arousal pair of happiness and sadness than for the low arousal pair of relaxed and tired. This was evident in the mid rather than early adolescence group. Adolescents tend to interact with more peers and are often exposed to more intense emotions arising from external and internal sources than children (Larson \& Sheeber, 2008) and such factors could increase an understanding of this intense co-occurring experience. This evidence supports trends found in adult populations that the intensity of the arousal as well as the valence of the specific emotion pairs impacts on the type of simultaneous mixed emotions experienced (Ojeca \& Carrera, 2009; Rafaeli et al., 2007). The findings may show a developmental trend from early to mid-adolescence in the differentiation of experiences by the intensity of the emotion pair. It could be argued that by mid adolescence there have been more opportunities to reflect on and identify experiences in relation to intensity. It could also be argued that increasing self-reflective capacities (Adams-Webber, 1999; Lyons \& Zelazo, 2011) and increasing complexity in conceptual representations, including the 
abilities to think abstractly and combine abstract ideas (e.g., Yong-Ju \& Lawson, 2000; Yurgelun-Todd, 2007), across this age range relate to the ability to report more complex simultaneous mixed emotion experiences in relation to varying intensity. The ESM is consistent with reported simultaneous experiences in relation to opposite valence high and low intensity emotions, whereas the CM would not afford reported experiences in relation to such opposite valence experiences of contrasting intensity

It is of note that no effects of emotion pair, considerations of self and other or age group emerged for the prevalence mixed emotion experience. Having two emotions occurring at the same time with constant relative levels of intensity (e.g., very high happiness and very low sadness) throughout the experience may be more difficult to detect in oneself and others. This may be because the intensity of each emotion does not switch across the duration of the experience and may be related to no activation of a switch in attention.

The study is not without limitations. A manipulation check to ensure that participants were considering themselves or another child would improve confidence in their focus during administration of the vignettes. A physiological measure of affective activation was not included to check genuine activation rather than affective representations of the target emotions. It could be argued that the pairing of happiness and sadness is not directly contrasting in intensity with relaxation and tiredness, although the pairs are argued to be of contrasting intensity of arousal to some extent (Larsen \& McGraw, 2014). A measure of how participants regard this contrast would attest to the validity of the choice of the extent of the contrasting intensity of these emotion pairings. The emotion pairs may 
differ in their type beyond levels of arousal. For example happiness and sadness are of a more basic nature (Heubeck et al., 2015) than relaxation or tiredness which may represent more nuanced emotional experiences. Such influences of emotion types on simultaneousness mixed emotion experiences beyond arousal intensity could be assessed in future work.

Importantly, the patterns of types of mixed emotion experiences uncovered in this study, the associations with adolescents' own mixed emotion experience, and the understanding of the mixed emotion experience of others should be further explored. The emotion pairings could be adapted to increase the ambivalence resulting from the conflict between co-occurring opposite affects (Braniecka, Trzebińska, Dowgiert, \& Wytykowska, 2014). The protagonist in the other condition and the mixed emotion events could be altered to increase task relevance and the potential emotional impact with the addition of repeat stimulus trials. Research exploring emotion recognition and experiences between genders could also be conducted to explore the view that males and females differ in their understanding and processing of the social world with females often interpreting experiences more intensely than males (Grossman \& Wood, 2003).

A longitudinal study of mixed emotion experiences across early childhood to early adulthood varying emotion pairs (Heubeck et al., 2015), protagonists (Burkitt \& Watling, 2015), and events (e.g., those which provoke real life mixed emotions; Ojeca \& Carrera, 2009) could shed light on the developmental pathways for the complex emotion processing of sequential and simultaneous mixed emotion experiences throughout adolescence and examine how this might relate to the development of emotional skills such as emotion 
regulation (Larson \& Sheeber, 2008). Models of emotion development (Pons, Harris, \& de Rosnay's, 2004; Rocha et al., 2015) could be extended to consider how the recognition and understanding of simultaneous types of mixed emotion in self and other proceeds through adolescence and explore the antecedents of this development. Research could assess how the AES might complement current emotion assessment tools (Viljoen, Shaffer, Gray, \& Douglas, 2017) by examining experiences and changes of more than one emotion across time in educational, clinical and forensic settings.

Overall, the present research has demonstrated for the first time that adolescents identify sequential and simultaneous mixed emotions of the four types varying in intensity and temporal patterns as those identified in adult and tentatively in childhood populations. The findings attest to the utility of the AES measure within adolescent populations and, indicate the possibility of extending developmental models of subjective simultaneous mixed emotion experience to consider the emotional intensity of simultaneous mixed emotion experiences across this age range for emotions with both high and low arousal levels. 


\section{References}

Adams-Webber, J. (1999). Self-reflection in adolescence. Journal of Constructivist Psychology, 12(3), 203-213. doi: 10.1080/107205399266073

Berrios, R., Totterdell, P., \& Kellett, S. (2015). Eliciting mixed emotions: A meta-analysis comparing models, types, and measures. Frontiers in Psychology, 6, 428. doi:10.3389/fpsyg.2015.00428

Braniecka, A., Trzebińska, E., Dowgiert, A., \& Wytykowska, A. (2014). Mixed emotions and coping: The benefits of secondary emotions. Plos ONE, 9(8), e103940. doi: 10.1371/journal.pone.0103940

Burkitt, E., Lowry, R. G., \& Fotheringham, F. (2017). Children's understanding of mixed emotions in self and other: Verbal reports and visual representations. Infant and Child Development. doi 10.1002/icd.2076

Burkitt, E., \& Watling, D. (2015). How do children who understand mixed emotion represent them in freehand drawings of themselves and others? Educational Psychology, 36(5) 935-955. doi:10.1080/01443410.2015.1044942

Cacioppo, J. T., Gardner, W. L., \& Berntson, G. G. (1999). The affect system has parallel and integrative processing components: Form follows function. Journal of Personality and Social Psychology, 76, 839-855. doi:10.1037/0022-3514.76.5.839

Carrera, P., \& Oceja, L. (2007). Drawing mixed emotions: Sequential or simultaneous experiences? Cognition and Emotion, 21(2), 422-441.

doi:10.1080/02699930600557904 
Fang, X., Sauter, D. A, \& Van Kleef, G. A. (2017). Seeing mixed emotions: The specificity of emotion perception from static and dynamic facial expressions across cultures. Journal of Cross Cultural Psychology, 49(1), 130-148. doi: $10.1177 / 0022022117736270$

Faul, F., Erdfelder, E., Lang, A.-G., \& Buchner, A. (2007). G*Power 3: A flexible statistical power analysis program for the social, behavioral, and biomedical sciences. Behavior Research Methods, 39, 175-191. doi: 10.3758/BF03193146

Grossman, M., \& Wood, W. (1993). Sex differences in intensity of emotional experience: a social role interpretation. Journal of Personality and Social Psychology, 65(5), 1010-1022. doi:10.1037/0022-3514.65.5.1010

Heubeck, B. G., Butcher, P. R., Thorneywork, K., \& Wood, J. (2015). Loving and angry? Happy and sad? Understanding and reporting of mixed emotions in mother-child relationships by 6- to 12-year-olds. British Journal of Developmental Psychology, 34(2), 245-260. doi:10.1111/bjdp.12128

Larsen, J. T., \& McGraw, A. P. (2014). The case for mixed emotions. Social and Personality Psychology Compass, 8(6), 263-274. doi:10.1111/spc3.12108

Larsen, J. T., McGraw, A. P., \& Cacioppo, J. T. (2001). Can people feel happy and sad at the same time? Journal of Personality and Social Psychology, 4, 684-696.doi: $10.1037 / / 0022-3514.81 .4 .684$ 
Larson, R. W., \& Brown, J. R. (2007). Emotional development in adolescence: What can be learned from a high school theater program? Child Development, 78(4), 1083-1099. doi: $10.1111 / \mathrm{j} .1467-8624.2007 .01054 . x$

Larson, R. W., \& Sheeber, L. B. (2008). The daily emotional experience of adolescents: Are adolescents more emotional, why, and how is that related to depression? In N. B. Allen \& L. B. Sheeber (Eds.), Adolescent emotional development and the emergence of depressive disorders (pp. 11-32). Cambridge University Press. doi: 10.1017/CBO9780511551963.002

Larson, R. W., Moneta, G., Richards, M. H., \& Wilson, S. (2002). Continuity, stability, and change in daily emotional experience across adolescence. Child Development, 73(4), 1151-1165. doi: 10.1111/1467-8624.00464

Lyons, K. E., \& Zelazo, P. D. (2011). Monitoring, metacognition, and executive function: Elucidating the role of self-reflection in the development of self-regulation. Advances in Child Development and Behavior, 40, 379-413. doi: 10.1016/B978-0$12-386491-8.00010-4$

Norris, C. J., Gollan, J., Berntson, G. G., \& Cacioppo, J. T. (2010). The current status of research on the structure of evaluative space. Biological Psychology, 84(3), 422436. doi:10.1016/j.biopsycho.2010.03.011.

Oceja, L., \& Carrera, P. (2009). Beyond a single pattern of mixed emotional experience: Sequential, prevalence, inverse, and simultaneous. European Journal of Psychological Assessment, 25(1), 58-67. doi:10.1027/1015-5759.25.1.58 
Pons, F., Harris, P. L., \& de Rosnay, M. (2004). Emotion comprehension between 3 and 11 years: Developmental periods and hierarchical organization. European Journal of Developmental Psychology, 1(2), 127-152. doi:10.1080/17405620344000022

Rafaeli, E., Rogers, G. M., \& Revelle, W. (2007). Affective synchrony: Individual differences in mixed emotions. Personality and Social Psychology Bulletin, 33(7), 915-932. doi:10.1177/0146167207301009

Rocha, A., Roazzi, M., Lopes da Silva, A., Candeias, A., Moita Minervino, C., Roazzi, M., \& Pons, F. (2015). Test of Emotion Comprehension: Exploring the underlying structure through Confirmatory Factor Analysis and Similarity Structure Analysis. In A. Roazzi, B. C. de Souza \& W. Bilsky (Eds.), Facet theory: Searching for structure in complex social, cultural and psychological phenomena (pp. 66-84), UFPE. doi: 10.13140/RG.2.1.2457.4483

Russell, J. A., \& Carroll, J. M. (1999). On the bipolarity of positive and negative affect. Psychological Bulletin, 125(1), 3-30. doi:10.1037/0033-2909.125.1.3

Smith, J. P., Glass, D. J., \& Fireman, G. (2015). The understanding and experience of mixed emotions in 3-5 year-old children. The Journal of Genetic Psychology, 176(2), 65-81. doi10.1080/00221325.2014.1002750

Viljoen, J. L., Shaffer, C. S., Gray, A. L., \& Douglas, K. S. (2017). Are adolescent risk assessment tools sensitive to change? A framework and examination of the SAVRY and the YLS/CMI. Law and Human Behavior, 41(3), 244-257. doi:10.1037/lhb0000238. 
Wahlstrom, D., Collins, P., White, T., \& Luciana, M. (2010). Developmental changes in dopamine neurotransmission in adolescence: Behavioral implications and issues in assessment. Brain and Cognition, 72(1), 146. doi: 10.1016/j.bandc.2009.10.013

Wintre, M., \& Vallance, D. D. (1994). A developmental sequence in the comprehension of emotions: Intensity, multiple emotions, and valence. Developmental Psychology, 30(4), 509-514. doi: 10.1037/0012-1649.30.4.509

Yong-Ju, K., \& Lawson, A. E. (2000). Linking brain growth with the development of scientific reasoning ability and conceptual change during adolescence. Journal of Research in Science Teaching, 37(1), 44-63. doi: 10.1002/(SICI)10982736(200001)

Yurgelun-Todd, D. (2007). Emotional and cognitive changes during adolescence. Current Opinion in Neurobiology, 17(2), 251-258. doi: 10.1016/j.conb.2007.03.009 


\section{Appendices}

Appendix A: Happy and Sad and Tired and Relaxed Vignettes for the Self and Other Condition.

\section{Self-condition vignettes}

Happiness and Sadness: You have just left your hometown and moved away with your family. You used to go to the local school with your close friend and you used to have fun together. You used to hang out in school and after school with your close friend. You will now not be able to hang out with your best friend which you used to enjoy. When you started at the new school there was no one to hang out with. After a while, you made two new friends from your class and you now hang out together. You often think about your friend from where you used to live and the new friends you have made.

Relaxed and Tired: You are playing tennis during the PE lesson at school. You enjoy tennis and the weather is nice so it is an enjoyable game. When the first set it over you are looking forward to sitting down for 5 minutes. The next set is a close set and you are out of breath from working hard. You and your partner are having fun playing tennis as you both enjoy the game. You have won the game and you are pleased you have been able to play. You and your partner are ready to go home, sit down and enjoy a carefree evening thinking about the game. 


\section{Other condition vignettes}

Happy and sad condition: Alex has just left his/her hometown and moved away with his/her family. Alex used to go to the local school with his/her close friend and they used to have fun together. Alex used to hang out in school and after school with his/her close friend. Alex will now not be able to hang out with his/her best friend which he/she used to enjoy. When Alex started at the new school there was no one to hang out with. After a while, Alex made two new friends from his/her class and they now hang out together. Alex often thinks about his/her friend from where he/she used to live and the new friends h/she has made.

Relaxed and tired condition: Alex is playing tennis during the PE lesson at school. Alex enjoys tennis and the weather is nice so it is an enjoyable game. When the first set it over Alex is looking forward to sitting down for 5 minutes. The next set is a close set and Alex is out of breath from working hard. Alex and his/her partner are having fun playing tennis as they both enjoy the game. They have won the game and Alex is pleased they have been able to play. Alex and his/her partner are ready to go home, sit down and enjoy a carefree evening thinking about the game. 
Appendix B: Example Analogue Emotion Scale

Participants were asked to think about their condition appropriate (own or Alex's) emotional experience at the end of the vignette and use the following graph where the happy / sad or relaxed / tired condition appropriate words were included and their own or Alex's feelings were referred to. The key was explained and they could represent the emotions in either order.

Key:

A straight line __ happy (or relaxed)

A dotted line ......................sad (or tired)

(How much (you/they) felt)

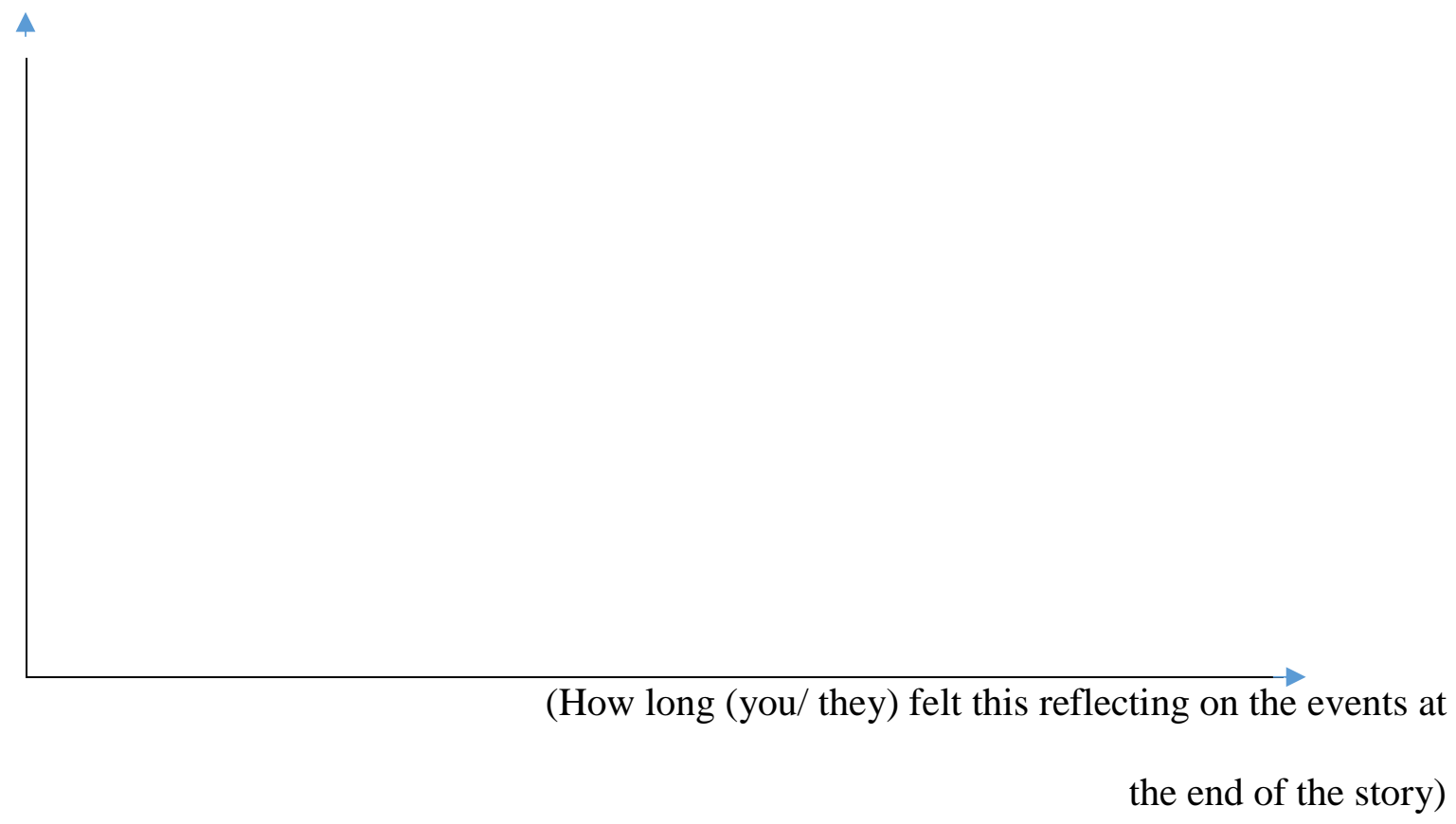

Figure B1. Example AES graph 